\title{
Penetrating Cardiac and Great Vascular Injuries in Wars, Treatment Algorithm Improve the Outcomes and Keep People's Life
}

\author{
Hadi abdulsalam Abo Aljadayel ${ }^{* 1}$, Tameem Azzawi ${ }^{2}$, Hussein Alkanj ${ }^{3}$, Fadi Butruss ${ }^{4}$, \\ A.SalahKoja ${ }^{5}$ \\ ${ }^{I}$ Abo Aljadayel, Master Cardiac Surgery, Syrian and Arab Board Cardiac Surgery \\ ${ }^{2}$ Master in Thoracic Surgery \\ ${ }^{3}$ DIS In Thoratic Cardiovascular Surgery, Nancy; Hasan Aslan, MD, Syrian Board Cadrdiac Surgery \\ ${ }^{4}$ Subspecialist in Cardiovascular and Thoracic Anest \\ ${ }^{5}$ A.Salahkoja, MD, AFSA, DU Cardiovascular Surgery
}

*Corresponding Author: Hadi abdulsalam Abo Aljadayel, Abo Aljadayel, Master Cardiac Surgery, Syrian and Arab Board Cardiac Surgery

\begin{abstract}
Purpose of the Study: we found that treatment algorithm improve the work and keep people's life after gunshots and explosions.
\end{abstract}

Methods: Retrospective analysis of a group of a total of 24 patients who underwent a surgery for war penetrating cardiac and great vessels injury since 7/2012 to 4/2015. Simple chest X ray (CXR), Abdominal X ray $(A X R)$, the suspect and high possible course of the gunshot, simple $2 M$ echo Philips just to distinguish if there pericardial effusion or no, and if suspect we follow the rule "open and see don't wait and see".

Results: The mean age of the patients included in the group was $33.5 \pm 33.1$ years, with men significantly prevailing (19 patients, $79.1 \%)$. A total of 3 injured persons (12.5\%) were haemodynamically stable when admitted, 2linjured persons (87.5\%) were unstable or in critical condition. All the penetrating cardiac and great vessels injuries were repaired by cardiac surgeon. The injury of coronary arteries was in one case managed by ligation of the peripheral part of the coronary artery. In 14 patients (58.3\%) also a penetrating injury of other organs was simultaneously managed. The mean ICU stay was two days except for complicated cases, on average $4 \pm 9$ units of red blood cells were administered during the in-hospital stay which lasted on average $7.1 \pm 2.4$ days.

Discussion: A cardiac arrest, severe hemodynamic instability, unconsciousness, serious concomitant injury, gunshot injury, multiple injuries represent predictors of death in these injuries. The total mortality in penetrating cardiac and great vessels injury ranges from 16 to $25 \%$, the presence of vital signs at the time of hospital admission is associated with 75-84\% probability of survival. The surviving patients show excellent short-term results with the exception the case who suffered neurologic complication (4.1\%)

Conclusions: Our experience proves a high survival rate of patients with penetrating war cardiac and great vessels injury. Treatment algorithm and a vital interdisciplinary cooperation are the key goal of successful management of these injuries. 'Open and see don't wait and see' is the rule which should be follow. No need to lost time with more investigations if there is doubt.

Keywords: penetrating war injury, cardiac injury, great vessel injury, outcome.

\section{INTRODUCTION}

Penetrating cardiac traumas are rarely seen and life-threatening clinical situations. Rapid and correct diagnose and intervention is important. Although prognosis and outcome is often not good in cardiac gunshot injuries $(1,13,14)$, cardiac surgeons still try to do more succeed. In the early 18th century Boerhaeve labeled all penetrating cardiac trauma as fatal (1). Billroth stated "The surgeon who should attempt to suture a wound of the heart should lose the respect of his colleagues" (11). Rehn succeeded in repairing a $1.5 \mathrm{~cm}$ right ventricular stab wound procedure named cardiorraphy (12). Hill 
did the first successful cardiorraphy in the USA (8).

Gunshot and bullets injuries are one of the penetrating traumas of the heart and that require urgent intervention $(1,2,5)$, Localization of the cardiac injury, rapid diagnosis and surgical intervention is life-saving in such patients $(1,6,10)$ and is important in terms of appropriate treatment approach.

True incidence of cardiac trauma in the military is difficult to ascertain. On the battle field many cardiac injuries are fatal. Dixon and McEwen in 1916 proclaimed, "Probably nearly all cardiac wounds produce death from haemorrhage too quickly to allow the patient being removed alive even to a short distance from the battle field $(1,13)$.

The most common chamber involved in projectiles injuries of the heart is the right ventricle obviously due to its anatomical location. Most of the series conclude that the right ventricle is the most common chamber involved $(1,9)$.

Frequently, the dominant physiological injury in surviving patients is cardiac tamponade. Cardiac tamponade provides an early opportunity for survival; however, it also contributes very rapidly to mortality associated with cardiac wounds $(1,5)$.

In our study, we present a surgical intervention for 24 patients who had cardiac injury due to gunshot or bullets in Aleppo University Hospital between $1^{\text {st }}$ of July 2012 and $30^{\text {th }}$ April 2015.

\section{Pre CASES INFORMation}

In Aleppo University Hospital which is belonged to Aleppo University when the war in Syria started the surgical department had meeting and putted treatment algorithm to improve the work and to keep people life after gunshots and explosions as possible from this rules some related to cardiac surgery which are:

- All cases which had suspect for mediastinum injuries must evaluate from cardiac surgeon.

- All cardiac surgery residents must knew how to do simple echo for the patients and distinguish pericardial effusion and underwent short coc ursein echocardiography department for that.

- For stable patients, chest CT scan for patients must done every time there suspect in mediastinum injury.
- All patients with unstable hemodynamics must resuscitate with fluid and transferred to operation room directly.

- The expected course of the gunshot or the signs of tamponed or shock are indication for direct exploration without investigations.

- We follow the rule "open and see don't wait and see" in high suspected stable patients with sup xiphoid incision to explore the pericardium.

- Leave every foreign body if it doesn't cause complications.

- All patients with gunshot should have three vein cannulas in three big veins and sample should sent directly to laboratory for blood group and cross Mach.

- Fluid must start immediately and as the needed and the hemodynamic situation.

- All patients with surgical procedures should take spread spectrum of antibiotics.

- All patients with heart injuries must follow up from cardiologists and cardiac surgeon and anesthesiologists and must underwent echocardiography before discharge and 15 days post discharge from hospital and every time needed (10).

\section{MeTHODS}

In our hospital we depends on simple chest $X$ ray (CXR), Abdominal $\mathrm{X}$ ray (AXR), the suspect and high possible course of the gunshot,simple2M echo Philips just to distinguish if there pericardial effusion or no, and if suspect we follow the rule "open and see don't wait and see".

Every time we used $U$ shape stitches for ventricular injuries supported by pericardial bled get and continuous simple suturing for atrial injuries. All patents underwent echocardiography and electrocardiography (ECG) post operation and 15 days after discharge from hospital and every time needed $(3,4)$.

\subsection{Definitions}

We considered and defined abnormal pericardium findings if there is any pericardial effusion more than $5 \mathrm{~mm}$ or any density beside one or more of the cardiac walls seen with echo (3).

\subsection{The cases:}

- 23 y old man brought to emergency room unstable hemodynamic BP 80/40 P=132/m 
examination seen entrance hole in the right inferior costal edge at the anterior axillary line. CXR revealed metal body in the mediastinum. The patient transferred directly to operation room for laparotomy and the general surgeon had found liver wound and wound in the diaphragm so consulted cardiac surgeon. We extend the abdominal incision upward and examined the pericardium and found full thickness 1 $\mathrm{cm}$ long wound in the inferior wall of right ventricle which sutured by polyprolene $3 / 0$ $\mathrm{U}$ shape tow stiches. The patient stayed in surgical ICU (Intensive Care Unit) for two days, weaned from ventilation in the next day of operation and then transferred to ward, the drains removed in the fourth day and he discharged home in the sixth day with good situation. Post operation: Echocardiography, ECG, was normal.

- During his stay in hospital 5 blood units, 10 units of FFP (Fresh Frozen Plasma), 12 units of platelets was administered.

- After 15 days of discharge the patient was in good situation with good wound heal and there were no complications, returned to his work after 45 days of operation.

- 50 years old man entered the emergency room with shock $\mathrm{BP}=60 / 30 \mathrm{P}=150 / \mathrm{m}$ he had entrance hole in the supra xiphoid notch and exit hole in the left costal edge in the anterior axillary line abdominal echo showed that there is no free fluid in the abdomen. the patient transferred directly to operation room, anterolateral thoracic incision was made and we found about $7 \mathrm{~mm}$ wound in the right ventricle which sutured by polyproprolene $3 / 0 \mathrm{u}$ shape 2 stiches supported with pericardium and the patient transferred to surgical ICU weaned from ventilation in the next day and was followed by general surgeon in the fourth day the patient developed severe abdominal pain and high temperature, in the abdominal echo there was free fluid so he underwent laparotomy and they found hole in the transverse colon and peritonitis after operation the patients transferred to ICU and he dead after about 6 hours because of shock mostly septic shock.

- 66 years old man brought to the emergency room after explosion he has many wounds on his chest and many bullets in the chest on CXR, abdomen and left knee. $\mathrm{BP}=90 / 50$ $\mathrm{p}=100$, abdominal echo shows free fluid, thoracic CT showed pericardial effusion, bleeding from knee incision. We opened mid sternotomy and we found hole in the right ventricle outlet which repaired with polypropolene $3 / 0 \quad \mathrm{U}$ shaped stiches supported with pericardial bled gets. General surgeon found multi intestinal injuries which repaired, vascular surgeon found injury in the popliteal vein repaired with saphenous vein. The patient post operation has oozing from all incisions and nonsurgical bleeding as the exploration hadn't reveal any surgical source for bleeding with about 13 units of blood, 20 units of FFP, 24 units of platelets was administered without any response and he dead the next day of surgery mostly hypovolemic shock and DIC (diffuse intra vascular coagulopathy).

- 22 years old man brought to the emergency room after gunshot $\mathrm{BP}=100 / 60 \mathrm{P}=110$ there was entrance hole in the costal edge near the anterior axillary line and exit hole in the left epigastria after abdominal echo the patient underwent laparotomy and after the general surgeon repaired liver wound and did spleenectomy he saw blood coming from mediastinum to the abdomen he called us, we extended the incision upward to the lower part of sternum and open the pericardium to find right ventricle injury with injury to mid part of acute marginal artery we legated the artery and sutured the right ventricle with polypropolene 3/0 U shape stiches. The patient went to surgical ICU was stable and in hospital stay 4 units of blood, 6 units of FFP, and 6 units of platelets was administered, weaned from ventilation next day went toward after 2 day and removed the drainages in the $6^{\text {th }}$ day discharged to home in the $7^{\text {th }}$ day. Came back for checkup 15 days later echocardiography was normal, good wound healing, good hemodynamics return to his normal work 45 days after operation.

- 32 years old lady entered to emergency room after explosion with many wounds in the chest $\mathrm{BP}=90 / 50, \mathrm{P}=110$ with congestion in the jugular veins CXR revealed 3 small bullets in the mediastinum, abdominal echo said no free fluid, we saw the patients and with echo we saw pericardial effusion so the patient transferred to operation room we decided to open anterolateral left 
thoracotomy there was clots and black blood in the pericardium, we found about $3 \mathrm{~mm}$ hall in the right atrium sutured directly with polypropolene 3/0. In surgical ICU the patient was stable and weaned from ventilation next day and went to the ward the day after there was no need to transfer any blood derived and she discharged home after 6 days also she had normal echocardiography and wound heal at 15 days follow up.

- 30 years old man brought to the emergency after explosion he was anxious $\mathrm{BP}=130 / 70$ $\mathrm{P}=88$ and said that feel pain in the right shoulder there was small wound at the upper edge of scapula without any another wound had seen, CXR reveal foreign body in the mediastinum, chest tube inserted to right pleura and about 400cc of blood exit, after about one hour the patient developed tachycardia and hypotension $\mathrm{BP}=90 / 40$ $\mathrm{P}=130$ emergent echo for the pericardium revealed effusion and the patient transferred to operation room and we opened anterolateral incision in the left side, there was hale in the right ventricle outlet we sutured with polypropolene $3 / 0$ and we found the bullet in the inferior wall of the heart sup epicardium we put $U$ shape stich with pericardial bled get around it and opened the epicardium to remove it. The patient stay in ICU for two days without complication then transferred to ward and stay for 4 days then discharge to his home. The chest tube removed in the $5^{\text {th }}$ day he needed only 2 units of blood. The ECG, echocardiographies were normal in hospital and after 15 days also the clinical situation was good with good heal of the wound.

- 55 years old lady brought to emergency room with dyspnea and right chest pain examination $\mathrm{BP}=90 / 40 \mathrm{P} 120$ there was entrance hall in the right edge of the sternum $4^{\text {th }}$ intercostal space and exit hall sup right scapula, CXR revealed plural effusion and chest tube drained $1200 \mathrm{cc}$ the patient transferred to operation room and right $5^{\text {th }}$ intercostal space anterolateral incision achieved. There were 3 wounds in the right lung sutured with vicrile 0 and we notice that dark blood exit from opening in the pericardium we dilated this opening and found tear in the right appendage which sutured directly with polypropolene $3 / 0$. The patient stay in ICU for two days without complication then transferred to ward and stay for 6 days then discharge to his home. The chest tube removed in the $7^{\text {th }}$ day he 3units of blood was administered. The ECG, echocardiography was normal in hospital and after 15 days also the clinical situation was good with good heals of the wound.

- 16 years old young brought to emergency after gunshot there was entrance wound in the mid right clavicle without exit hole right plural chest tube drained $500 \mathrm{cc}$ and stopped $\mathrm{BP}=110 / 60 \mathrm{P} 110$. After 2 hours the patient had hypotension $\mathrm{BP}=80 / 40 \quad \mathrm{P}=140$ congested neck veins and chest $\mathrm{CT}$ scan revealed that the bullet was in the right ventricle, the patient directly transferred to operation room and opened trans $5^{\text {th }}$ intercostal space anterolateral incision before open the pericardium the patient developed cardiac arrest which responded to hand massage and drugs there was wide tear in the right atrium repaired by direct suturing polypropolene $3 / 0$. In surgical ICU the patient was stable, in hospital stay 3 units of blood and 3 units of FFP was administered, weaned from ventilation in the next day and transferred toward the day after and he discharged home after 6 days also he had normal echocardiography except for mild to moderate tricuspid valve regurgitation, at 15 days, 3 months follow up but the bullet kept at the right ventricle apex away from the septum.

- 12 years old boy brought to emergency after explosion with many small wounds in the anterior chest wall $\mathrm{p}=150 / \mathrm{m}$ CXR reveal many bullets in the chest and $1.5 \mathrm{~cm}$ pneumothorax in the right side bilateral chest tube inserted and about 200cc of blood exit from the right side only, emergent echo for pericardium reveal about $1 \mathrm{~cm}$ effusion so the child transferred to operation room and sup xiphoid incisions made there was about $100 \mathrm{cc}$ of dark blood and big about $3 * 3 \mathrm{~cm}$ clot removed there was erythematous patch $1 * 2 \mathrm{~cm}$ in the anterior surface of the right ventricle without bleeding we inserted drain and closed the incision, pulse $=110$ post operation the child weaned from ventilation 3 hours later there was no need to transfer any blood derived, went to ward next day removed the drain in the $6^{\text {th }}$ day and discharge in the $7^{\text {th }}$ day without any complication echocardiography and ECG were normal after 15 days also. 
- 26 years old lady came to emergency room after explosion with iterance wound in the right chest under the nipple $\mathrm{BP}=60 / 30$, $\mathrm{P}=145$, the patient transferred directly to operation room we decided to open anterior right thoracotomy there was wound in the middle and superior lopes of right lung and black blood exit from pericardium we opened the pericardium and found wound in the right atrium near atrioventricular groove sutured directly with polypropolene $3 / 0$, the lung wound also sutured with polypropolene 0 continuous with lock. In surgical ICU the patient stay for two days without complication, she took 4units of blood, 6 units of FFP weaned from ventilation next day and went to the ward the day after; she stayed for 6 days the chest tube removed in the 6th day, then discharge to home. The ECG, echocardiography was normal in hospital and after 15 days also the clinical situation was good with good heals of the wound.

- 46 years old man brought to emergency room unstable hemodynamic BP 80/40 $\mathrm{P}=132 / \mathrm{m}$ examination revealed entrance hole in the $5^{\text {th }}$ intercostal space at the edge of sternum. CXR, AXR revealed metal body in the abdomen under the liver. The patient transferred directly to operation room for laparotomy and the general surgeon found liver wound and wound in the right diaphragm so consulted cardiac surgeon. We extend the abdominal incision upward and to the right sixth intercostal space and examined the pericardia and found not full thickness $1 \mathrm{~cm}$ long wound in the anterior wall of right ventricle which sutured by polyprolene 3/0 U shape tow stiches with injury for small coronary artery which tied. The patient stayed in surgical ICU for two days, weaned from ventilation in the next day of operation and then transferred to ward, the drainage removed in the 5th day, he discharged in the sixth day with good situation. Post operation: Echocardiography, ECG, both were normal. During his stay in hospital he took 4 blood units, 6 units of FFP, 6 units of platelets. After 15 days of discharge the patient was in good situation with good wound heal and there were no complications.

- 28 years old man brought to emergency room with unstable hemodynamic BP 80/40 $\mathrm{P}=140 / \mathrm{m}$ examination seen entrance hole in mid sternum and exit wound in the posterior near first right lumber section. The patient transferred directly to operation room for laparotomy and the general surgeon had found liver, gastric wounds and wound in the diaphragm so consulted cardiac surgeon. We extend the abdominal incision upward and to the left intercostal space and examined the pericardia and found full thickness $1 \mathrm{~cm}$ long wound in the anterior wall of right ventricle which sutured by polyprolene 3/0 U shape tow stiches. The patient stayed in surgical ICU for two days, weaned from ventilation in the next day of operation and then transferred to ward, the drainage removed in the 5th day, he discharged in the sixth day with good situation. Post operation: Echocardiography, ECG were normal. During his stay in hospital he took 6 blood units, 8 units of FFP, 6 units of platelets. After 15 days of discharge the patient was in good situation with good wound heal and there were no complications.

- 24 years old young brought to emergency after gunshot there was entrance wound in the right intercostal space at the edge of sternum without exit hole right plural chest tube drained 200cc and stopped $\mathrm{BP}=80 / 40$ P140 CXR reveals bullet in the mediastinum, the patient directly transferred to operation room and opened midsternotomy incision there was big clot about $7 * 10 * 1 \mathrm{~cm}$ and a wound in the right ventricle near mid part of LAD which sutured with polypropolene $3 / 0 \mathrm{U}$ shape stiches passes under the LAD artery supported with pericardial bled get. In surgical ICU the patient was stable, he took 2 units of blood and 2 units of FFP, weaned from ventilation in the next day and transferred toward the day after and he discharged home after 6 days also he had normal ECG echocardiography at 15 days.

- 30 years old man brought to the emergency after explosion he was anxious $\mathrm{BP}=90 / 50$ $\mathrm{P}=120$ and said that feel pain in the right chest there was small wound in the third intercostal space without any another wound had seen, CXR reveal foreign body in the mediastinum, chest tube inserted to right pleura and about $300 \mathrm{cc}$ of blood exit. echo for pericardium reveal effusion so the patient transferred to operation room and we opened right $5^{\text {th }}$ intercostal anterolateral 
incision, there was hole in the anterior surface of the right ventricle we sutured with polypropolene $3 / 0$ and we found the bullet in the inferior wall of the heart sup epicardium and thin layer of myocardium of the left ventricle near PDA(posterior descending artery) artery we but U shape stich with pericardial bled get around which passed the PDA artery and opened the epicardium to remove it. The patient stay in ICU for two days without complication then transferred to ward and stay for 4 days then discharge to his home. The chest tube removed in the 5th day he took 2 units of blood, 4 units of FFP. The ECG, echocardiography were normal in hospital and after 15 days also the clinical situation was good with good heal of the wound.

- 18 years old man brought to the emergency after explosion he $\mathrm{BP}=80 / 40 \mathrm{P}=150$ and said that feel pain in the right chest there was small wound in the 4th intercostal space at the anterior axillary line without any another wound had seen, CXR reveal foreign body in the mediastinum, chest tube inserted to right pleura and about $1500 \mathrm{cc}$ of bright blood exit. the patient transferred to operation room and we opened right $5^{\text {th }}$ intercostal anterolateral incision, was wound in the middle and superior lopes of right lung and black blood exit from pericardium we opened the pericardium but we couldn't find and control the source of blood so we change the position of the patient to supine and opened the sternum we found wound in the left atrium we sutured with polypropolene $3 / 0$ and we found a bullet in the thymus only. The patient stay in ICU for two days without complication then transferred to ward and stay for 4 days then discharge to his home. The chest tube removed in the 6th day he took 6 units of blood, 8 units of FFP10 unites of platelets. The ECG, echocardiography was normal in hospital and after 15 days also the clinical situation was good with good heals of the wound.

- 40 years old man brought to the emergency after explosion he $\mathrm{BP}=90 / 40 \quad \mathrm{P}=130$ and said that feel pain in chest there was small wound in the 3th intercostal space at the sternal edge without any another wound had seen, CXR reveal foreign body in the inferior right pleura, chest tube inserted to right pleura and about $300 \mathrm{cc}$ of dark blood exit. The pericardial echo reveal effusion so the patient transferred to operation room and we opened the sternum we found a $1 \mathrm{~cm}$ wound in the anterior wall of right ventricle we sutured with polypropolene $3 / 0 \mathrm{U}$ shape supported with pericardium. The patient stay in ICU for two days without complication then transferred to ward and stay for 4 days then discharge to his home. The chest tube removed in the 6th day he took 3 units of blood, 2 units of FFP. The ECG, echocardiographies were normal in hospital and after 15 days also the clinical situation was good with good heal of the wound.

- 28 years old man brought to emergency room with shock and unstable hemodynamic BP 50/-- P>160/m, there was a lot off wounds all over his body. The patient transferred directly to operation room for laparotomy and the general surgeon had found many intestinal wounds but there was no abdominal bleeding to explain the shock so consulted cardiac surgeon. We extend the abdominal incision upward and to the left intercostal space and examined the pericardia and found clots and blood and there was full thickness $1 \mathrm{~cm}$ long wound in the anterior wall of right ventricle which sutured by polyprolene 3/0 U shape tow stiches and another wound in the inferior vena cava near connection to right atrium which sutured directly. The patient keep hypotension and then bradycardia and arrest without response to inotropes and hand massage we think because of deep shock.

- 55 years old lady came to emergency room after explosion with iterance wound in the right chest upper the nipple $\mathrm{BP}=80 / 30$, $\mathrm{P}=135$, right chest tube exit $900 \mathrm{cc}$ of blood, pericardial echo didn't reveal effusion then the patient transferred directly to operation room we decided to open anterior right thoracotomy there was wound in the middle lope of right lung and black blood exit from pericardium we opened the pericardium and found wound in the right atrium mid lateral surface sutured directly with polypropolene $3 / 0$, the lung wound also sutured with polypropolene 0 continuous with lock we didn't found the bullet. In surgical ICU the patient stay for two days without complication, she took 4units of blood, 6 unitsof FFP, 6 units of platelets, weaned 
from ventilation next day and went to the ward the day after, she stayed for 6 days The chest tube removed in the 6th day, then discharge to home. The ECG, echocardiographies were normal in hospital and after 15 days also the clinical situation was good with good heal of the wound.

- 23 years old man brought to emergency room with shock and unstable hemodynamic BP 50/-- P>160/m, entrance wound in the right side $2 \mathrm{~cm}$ above the nipple and exit wound in the $5^{\text {th }}$ left intercostal space at mid axillary line. The patient transferred directly to operation room we entered lateral left $5^{\text {th }}$ intercostal space, there was a lot of blood in the plural cavity and the pericardium there was small Halle in the anterior surface of the right ventricle and more than $1 \mathrm{~cm}$ serrate edges in the lateral surface of left ventricle we sutured both of them by polyprolene 3/0 U shape tow stiches. The patient keep hypotension and then developed bradyca21 ZDrdia and distend of the right ventriclethen arrest without response to inotropes and hand massage we think because of deep shock and ventricular septum defect.

- $\quad 3.5$ years old girl brought to emergency after explosion with many small wounds in the anterior chest and abdominal wall $\mathrm{p}=150 / \mathrm{m}$ CXR revealed many small wounds in the chest, bilateral chest tube inserted and less than 50cc of blood exit from both sides, emergent echo for pericardium reveal about $1 \mathrm{~cm}$ effusion. Abdominal echo there was free fluid the child transferred to operation room for laparotomy and there was liver wound which sutured then we extended the abdominal incision to sup xiphoid incision to check the pericardium there was about 25 cc of dark blood and big about $1 * 2 \mathrm{~cm}$ clot removed there was erythematous patch $1 * 1$ $\mathrm{cm}$ in the anterior surface of the right ventricle without bleeding we inserted drain and closed the incision, the child weaned from ventilation next day, went to ward next day removed the drain in the $6^{\text {th }}$ day and discharge in the $7^{\text {th }}$ day from cardiac surgery follow up without any complication echocardiography and ECG were normal before discharge and after 15 days also.

- 19 years old man brought to the emergency after explosion he $\mathrm{BP}=90 / 40 \quad \mathrm{P}=130$ and said that feel pain in chest there was small wound in the right 3 th intercostal space at the sternal edge without any another wound had seen, CXR reveal foreign body in the mediastinum, chest tube inserted to right pleura and about $100 \mathrm{cc}$ of dark blood exit. The pericardial echo reveal effusion, the neck veins were congested so the patient transferred to operation room and we opened the anterolateral left intercostal space we found a $3 \mathrm{~cm}$ pericardial effusion and big clot in front of right ventricle when we removed it there was a wound in the anterior wall of right ventricle we sutured with polypropolene $3 / 0 \mathrm{U}$ shape supported with pericardium. The patient stay in ICU for two days without complication then transferred to ward and stay for 4 days then discharge to his home. The chest tube removed in the 6th day he took 2 units of blood, 2 units of FFP. The ECG, echocardiography were normal in hospital and after 15 days also the clinical situation was good with good heal of the wound.

- 45 years old lady brought to emergency after explosion there was many wounds in the chest and abdomen bilateral chest tube inserted but less than 200cc exit from each one $\mathrm{BP}=80 / 40 \mathrm{P} 140 \mathrm{CXR}$ reveals bullet in the mediastinum, abdominal echo said no free fluid in the abdomen, the patient directly transferred to operation room and opened midsternotomy incision there was big clot and a wound long in the right ventricle parallel to mid part of $\mathrm{LAD}$ (left descending artery) which sutured with polypropolene 3/0 U shape stiches passes under the LAD artery supported with pericardial bled get. In surgical ICU the patient was stable for the first 48 hours, he took 2 units of blood and 2 units of FFP, in the $3^{\text {rd }}$ day he developed abdominal pain with surgical abdomen transferred to operation room for laparotomy there was injury in the transverse colon the patient dead the next day of laparotomy.

- 70 years old man brought to emergency after explosion there was wounds in the mid sternum without exit wound $\mathrm{BP}=70 / 40 \mathrm{P} 140$ CXR reveals bullet in the mediastinum, fast abdominal echo said no free fluid in the abdomen, pericardial echo reveals effusion the patient directly transferred to operation room and opened midsternotomy incision there was big clot in the pericardium and about 300 blood we found wound in the 
right ventricle in the outlet sutured with polypropolene $3 / 0 \mathrm{U}$ shape stiches. The patient developed cardiac arrest which tack more than 12 mints to response and in the ICU he developed 3 times ventricular fibrillation responded to DC shock and the fourth time didn't respond and developed arrest and dead about 5 hours after operation.

- 23 years old man brought to the emergency after explosion he was anxious $\mathrm{BP}=70 / 40$ $\mathrm{P}=150$ and said that feel pain in the chest there was small wound in the right 4th intercostal space at the edge of sternum without any another wound had seen, CXR revealed foreign body in the inferior left pleura, chest tube inserted to left pleura and about 300cc of blood exit. echo for pericardium reveal effusion so the patient transferred to operation room and we opened left $5^{\text {th }}$ intercostal anterolateral incision, there was hale in the anterior surface of the right ventricle we sutured with polypropolene $3 / 0$, the patient developed heart arrest which responded after about continues 10 minutes of hand massage and adrenaline atropine. The patient stay in ICU for two days after weaned from ventilation he had aphasia and was disoriented, transferred to ward and stay for 12 days then discharge to his home. The chest tube removed in the 5th day he took 2 units of blood, 4 units of FFP. The ECG, echocardiography were normal in hospital and after 15 days also good heal of the wound, after 3 months he became neurologically better oriented with only heavy speech.

\section{DISCUSSION AND RESULTS}

Between $1^{\text {st }}$ July 2012 and $30^{\text {th }}$ April 2015 we had operated 24 patients who had gunshot or bullet or splinters in the heart. The mean age of the patients included in the study was $33.5 \pm$ 33.1 years, with men significantly prevailing (19 patients, $79.1 \%$ ). A total of 3 injured persons $(12.5 \%)$ were haemodynamically stable when admitted, 21injured persons (87.5\%) were unstable or in critical condition. All the penetrating cardiac and great vessels injuries were repaired by cardiac surgeon. From 24 patients $10(41.6 \%)$ had isolated heart injury two of them (20\%) dead, 4(16.6\%) patients had concomitant heart and lung injuries no one dead, $10(41.6 \%)$ had concomitant abdominal and heart injuries $4(40 \%)$ of them dead, seems that concomitant injuries increase the risk of death. From all 24 cases six patients were unlucky and dead for different reasons.

The first case dead because of deep shock which is due to the long time spent for transferring the patient from the field to the hospital (case number 17) The second was old man and maybe he had another cardiac injury so he didn't tolerate the hypotension and bleeding (case number 23).

The third had biventricular injury and surely VSD and we couldn't do something for him because we don't have cardio pulmonary bypass in emergency operation room (case 19).

The fourth and fifth dead weren't cardiac deaths but they were because of late discovering of abdominal injuries (cases 2, 22). The last death case was because of severe uncontrollable bleeding and diffuses intravascular coagulation and deep shock (case 3).

So there were four cardiac deaths $16 \%$ and six total deaths 25\%. Deep shock, cardiac arrest, concomitant injuries, left side injury, old age, were predictor factors for death. We had only one case $4.16 \%$ which had neurologic complication(case 24) and one patient has bullet in the right ventricle' one with mild to moderate tricuspid valve regurgitation (case 8).

We could remove the splinters in two cases $8.3 \%$ because it was sup epicardium and we believed that there was risk of bleeding and rupture of the heart and the splinters were under vision to leave it (cases 6,14 ).

We didn't found penetrating injury to the heart in two cases $8.3 \%$ but there was contusion in the right ventricle (cases 9, 20).

There was injury to coronary artery in one case $(4.16 \%)$ and the artery legated without any cardiac complication. The four cases with both heart and lung injuries the right chest was injured and we open right anterolateral thoracotomy in the $5^{\text {th }}$ intercostal space. All cases with abdominal injuries we choose to extend the abdominal incision upward and to the left $5^{\text {th }}$ intercostal space.

we did sternotomy only in $3(12.5 \%)$ when the patient had only mediastinal injury or we suspect aortic injury. we preferred left anterolateral thoracotomy if there is no indication to open sternum or right thoracic., all cases which arrived to the hospital alive the injury was in the right side of the heart except 
two cases, one in the left atrium and the second biventricular and ended with death.

The mean ICU stay was two days except for complicated cases, on average $4 \pm 9$ units of red blood cells were administered during the inhospital stay which lasted on average $7.1 \pm 2.4$ days. We couldn't knew the exact time which take for every case to arrive the emergency but we did the best to shorten the time inside the hospital, do less investigation, consult cardiac surgeon every time there doubt with meditational injury.

During this period and after we deal with this type of unusual injuries now we believe that:

- This type of injuries can be managed easily and heal completely if it was in the right side and when the patients arrived the hospital alive.

- The clinical situation and the course of the injury are the most important things which put indication for surgery at least to explore the pericardium.

With this plane we could control and manage the most cases of cardiac war injuries $84 \%$.

\section{CONCLUSION}

Our experience proves a high survival rate of patients with penetrating war cardiac and great vessels injury. treatment algorithm, and a vital interdisciplinary cooperation are the key goal of successful management of these injuries. 'Open and see don't wait and see' is the rule which should be follow and especially when there is suspect depends on the course of bullet or the hemodynamic status. Emergent CXR, and echo for pericardial effusion is easy, time saving and useful in many cases, no need to lost time with more investigations if there is doubt.

\section{REFERENCES}

[1] Wani ML, Ahangar AG, Wani SN, Irshad I, Ul-Hassan N. Penetrating Cardiac Injury: A Review. Trauma Mon. 2012; 17(1):230-2.7.

[2] Bali HK, Vijayvergia R, Binaries S, Kumar N. Gunshot Injury of the Heart: An Unusual Cause of Acute Myocardial Infarction.Tex Heart Inst J. 2003; 30(2):158-60.2.
[3] Harvey F, William F, Thomas R. Feigenbaums, 7th edition, 2010: 307-340.

[4] Kumar S, Moorthy N, Kapoor A, Sinha N. Gunshot Wounds Causing Myocardial Infaction, Delayed Ventricular Septal Defect, and Congestive Heart Failure. Tex Heart Inst J 2012; 39(1):129-32.

[5] Dawson J, Rodriguez Y, Pham SM, Ferreira A. Traumatic transection of the left anterior descending artery caused by a projectile. Journal of Cardiology Cases. 2012; 5(3):140-2.

[6] Kaya A, Caliskan E, Tatlisu MA, Hayiroglu MI, Tekkesin AI, Cakilli Y, et al. A Retained Bullet in Pericardial Sac: Penetrating Gunshot Injury of the Heart. Case Rep Cardiol. 2016; 2016:2427681.6.

[7] Hadi AA; Husssein A; Hasan A; Fadi B; Tameem A; A Salah K. Penetrating War Cardiac and Great Vessels Injury, Surgical Outcome Analysis in 24 Patients. Am J of Card and Cardiovas Disc. 2018; 1(2): 01-05.

[8] Olsovsky MR, Wechsler AS, Topaz O. Cardiac trauma. Diagnosis, management, and current therapy. Angiology 1997;48:423-32

[9] Galante J, London JA. Left Ventricular Bullet Embolus: A Case Report and Review of the Literature. J Emerg Med. 2010; 39(1):25-31.

[10] Harman PK, Trinkle JK. Injury to Heart. In: Moore EE, Mattox KL, Feliciano DV, editors. Trauma. 2nd ed. Norwalk: Appleton \& Lange; 1991.

[11] Crawford FA. Penetrating Cardiac injuries. In: Sabiston DC, editor. Textbook of surgery: the biological basis of modern surgical practice. 14th ed. Philadelphia: Saunders; 1991.

[12] Asensio JA, Murray J, Demetriades D, Berne J, Cornwell E, Velmahos G, et al. Penetrating cardiac injuries: a prospective study of variables predicting outcomes. J Am Coll Surg. 1998; 186(1):24-34. doi: 10.1016/S10 72-7515(97)00144-0.

[13] Trinkle JK, Marcos J, Grover FL, Cuello LM. Management of the wounded heart. Ann Thorac Surg. 1974; 17(3):230-6. doi: 10.1016/ S0003-4975(10)65641-X.

[14] Szentpetery S, Lower RR. Changing concepts in the treatment of penetrating cardiac injuries. J Trauma. 1977; 17(6):457-61. doi: 10.1097/00005373-197706000-00007.

Citation: Hadi abdulsalam Abo Aljadayel, Tameem Azzawi, et.al. Penetrating Cardiac and Great Vascular Injuries in Wars, Treatment Algorithm Improve the Outcomes and Keep People's Life. ARC Journal of Cardiology. 2020; 6(1): 18-26. doi:dx.doi.org/ 10.20431/2455-5991.0601005.

Copyright: () 2020 Authors. This is an open-access article distributed under the terms of the Creative Commons Attribution License, which permits unrestricted use, distribution, and reproduction in any medium, provided the original author and source are credited. 